\title{
Faults Diagnosis of Railway Bearing Based on FIR-wavelet Packet and LVQ Neural Network
}

\author{
Yang Jianwei ${ }^{1,2}$, Yao Dechen ${ }^{1,2,}{ }^{*}$, Li Xi $^{3}$, Jia Limin ${ }^{4,5}$ and Qin Yong ${ }^{4,5}$ \\ ${ }^{I}$ School of Machine-electricity and Automobile Engineering, Beijing University of Civil Engineering Architecture, Bei- \\ jing, 100044, China \\ ${ }^{2}$ Beijing Engineering Research Center of Monitoring for Construction Safety, Beijing University of Civil Engineering \\ Architecture, Beijing 100044, China \\ ${ }^{3}$ Subway Operation Technology Centre, Mass Transit Railway Operation Corporation LTD, Beijing 102208, China \\ ${ }^{4}$ State Key Laboratory of Rail Traffic Control and Safety, Beijing Jiaotong University. Beijing, 100044, China \\ ${ }^{5}$ Beijing Research Center of Urban Traffic Information Sensing and Service Technologies, Beijing Jiaotong University. \\ Beijing, 100044, China
}

\begin{abstract}
In this paper, we presented a way for railway bearing fault diagnosis with the use of FIR-wavelet packet and LVQ neural network. First, the original vibration signal of trains' rolling bearing is denoised based on FIR. Then, the signals after de-noised are preprocessed by wavelet packet and the wavelet packet energy eigenvector is reconstructed. Those kinds of wavelet packet energy eigenvectors are used to train LVQ neural network. Finally, the intelligent fault diagnosis is realized. The result shows that this approach is effective to distinguish this kind of rolling bearing faults. This method has important practical value.
\end{abstract}

Keywords: FIR, Wavelet packet, LVQ neural network, Railway rolling bearing, Fault diagnosis, De-noised.

\section{INTRODUCTION}

Roller bearings are key components in the railway sector. Therefore, fault diagnosis aimed at bearings has been the most important subject for extensive research.

There are many diagnosis methods for the roller bearing faults based on the vibration analysis, which have been successfully applied in some fields [1-3].

There are differences between other time frequency analysis method and wavelet packet analysis, the latter are advantageous over traditional methods in analyzing transient signals. With the help of wavelet transformation, the signal processing effect is similar to filtering through a band pass filter. As a result, it is possible to distinguish between the high frequency noise components, low frequency fault characteristic and resonance modulation components [4].

Lately, it is popular to use neural networks in nonparametric function learning because of their ability to learn complicated functions. It has been proved that multilayer back propagation networks can be used to approximate any continuous function [5].

*Address correspondence to this author at the School of Machine-electricity and Automobile Engineering, Beijing University of Civil Engineering Architecture, Beijing, 100044, P.R. China; Tel: +86 1051684773 ;

E-mail: shmily137@sina.com

\section{FIR}

FIR filter is used in signal processing due to its inherent advantages. FIR filter is straightforward to design and implement. It is always stable, and can correct the magnitude and phase responses, if desired [6].

Suppose the digital filter's input is $x(n)$, impulse response is $h(n)$,output is $y(n)$, then the mathematical expression is:

$y(n)=\sum_{k=0}^{n-1} h(k) x(n-k)$

Generally, the digital filter's frequency response may be represented as:

$H\left(e^{j w}\right)=\sum_{n=-\infty}^{\infty} h(n) e^{-j w n}$

FIR digital filter is the impulse response function for a finite number of values of the filter. Its frequency response can be expressed as:

$$
\begin{aligned}
& H\left(e^{j w}\right)=\sum_{n=0}^{N-1} h(n) e^{-j w n} \\
& H\left(e^{j w}\right)=H_{g}(w) e^{j \theta(\omega)}
\end{aligned}
$$

Where $H_{g}(\omega)$ is amplitude; $\theta(\omega)$ is phase. 


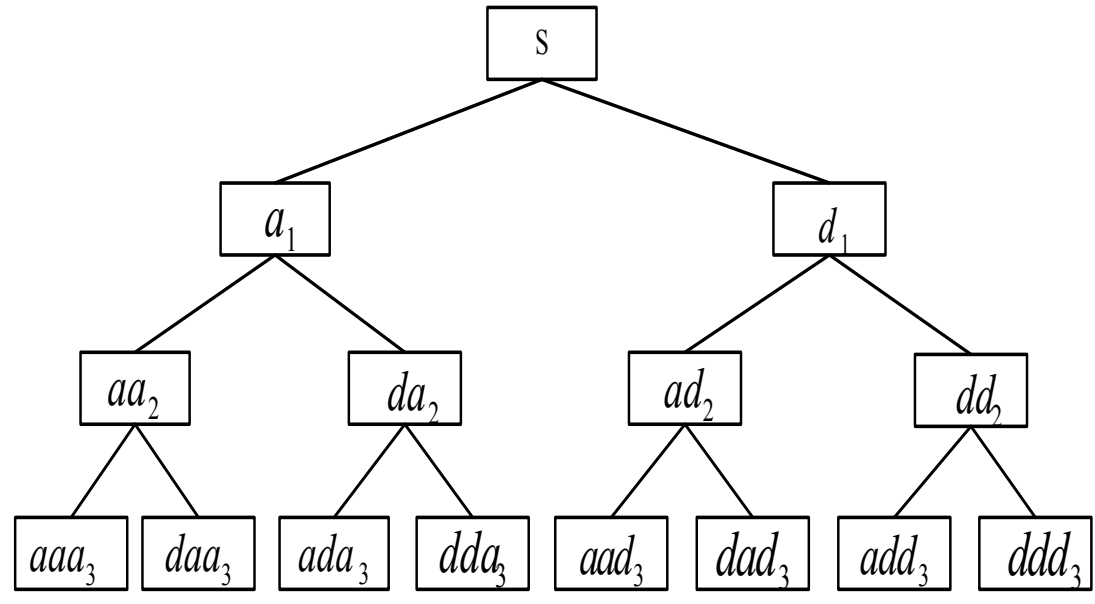

Fig. (1). Signal diagram of wavelet packet decomposition.

\section{WAVELET PACKET ANALYSIS}

Applying the wavelet transform to the original signal divides the signal into different frequency subbands so that each subband may be analyzed individually. A series of approximations is able to be obtained by reiterating such decompositions. The difference of the approximations between two successive decompositions is named after the details. The multi-resolution analysis (MRA) is a kind of algorithm which is based on the reiterative decomposition of the lowfrequency parts only. Also the peeling-off process in MRA is defined as decomposing of the approximation space $V_{j}$ into a subsequent approximation subspace $V_{j+1}$ and the corresponding detailed subspace $W_{j+1}$. The detailed space $W_{j}$ is related to the approximation space $V_{j}$, however, it is still under composed [7].

Wavelet packet transform is an extension of wavelet transformation achieved by approach of generalizing the link between multi-resolution approximation and wavelets. In wavelet packet transform, all the approximation space $V_{j}$ and the detailed space $W_{j}$ are decomposed even further. The transformation of the input sequence at scale $j$ can be described by:

$x_{0,0}(n)=x(n)$

$x_{j+1,2 l}(n)=\sum_{i=0}^{L_{2}-1} h_{1}(i) x_{j, l}\left(2^{j} i-n\right)$

$x_{j+1,2 l+1}(n)=\sum_{i=0}^{L_{2}-1} h_{0}(i) x_{j+1, l}\left(2^{j} i-n\right)$

where $h_{0}$ and $h_{1}$ represent low pass and high pass respectively having a finite-impulse response of size $K$.

When we aimed at the first level, we can decompose the signal into two subbands: low frequency sub-bands and high frequency sub-bands. When we turn to the next level, we can decompose the low frequency sub-bands into two parts, lower and higher frequency sub-bands. At the same time, the high frequency sub-bands are also decomposed into two parts, lower and higher frequency sub-bands (Fig. 1). The same decomposition goes on continually.

\section{WAVELET PACKET ENERGY VECTOR ALGO- RITHM}

(1) First, the signal was processed by three-layer wavelet packet. Fig. 1 shows the diagram result of wavelet decomposition, where $\mathrm{S}$ is used to represent original signal, $a_{1}$ is used to represent the 1 st low frequency coefficient $X_{10}$,which uses wavelet packet to decompose, $d_{1}$ represents the 1 st low frequency coefficient $X_{11}$, others is so on.

(2) The wavelet packet coefficient was constructed, then we take the signal characteristics of each band. $S_{30}$ represents the restructuring signal of $X_{30}, S_{31}$ represents the restructuring signal of $X_{31}$, others is so on, so the original signal $\mathrm{S}$ can be expressed:

$S=S_{30}+S_{31}+\cdots+S_{37}$

(3) The total energy of each band

$E_{3 j}=\int\left|S_{3 j}(t)\right|^{2} d t=\sum_{1}^{n}\left|x_{j k}\right|^{2}$

Where, $x_{j k}$ represents amplitude of the restructuring signal.

(4) The wavelet packet energy eigenvector was constructed

The definition of all the energy of signal:

$E=\sum_{j=0}^{7} E_{3 j}$ 


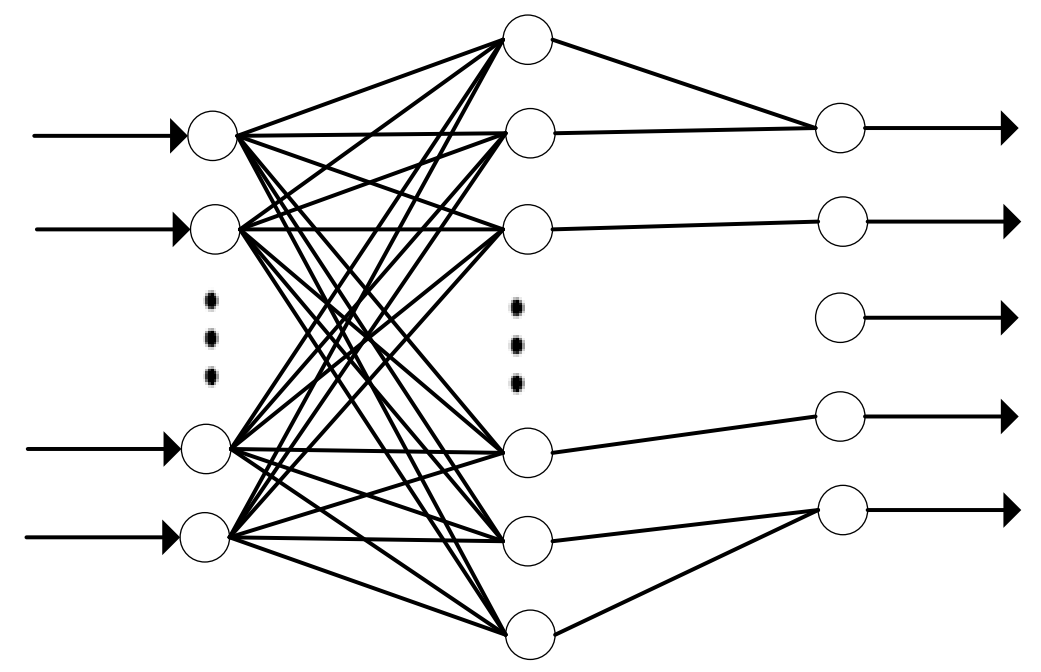

Fig. (2). LVQ structure.

A band of relative wavelet packet energy:

$p_{3 j}=\frac{E_{3 j}}{E}$

The definition of relative wavelet packet energy feature vector [8]:

$$
K_{i}=\left(p_{30}, p_{31}, \ldots, p_{37}\right)
$$

\section{LVQ NEURAL NETWORK MODEL}

Learning vector quantization is also seemed as a nearestneighbor pattern classifier based on competitive learning [910]. A LVQ network is composed of input layer, a Kohonen layer, which can be used to learn and perform the classification, and an output layer. The input layer is composed of one node for each input feature, the output layer is composed of one node for each class. Fig. (2) illustrates the structure of LVQ neural network.

When the LVQ processes the training, the Euclidean distance form a training vector, $x$, to each node's weight vector, $w_{i}$, in the Kohonen layer could be calculated according to the formula:

$d_{i}=\left\|w_{i}-x\right\|=\left\{\sum_{j=1}^{N}\left(w_{i j}-x_{j}\right)^{2}\right\}^{1 / 2}$

The nearest node is treated as the winner, and its weight vector is adjusted depending on whether the winning node is in the class of the training vector:

If the winner belongs to the correct class, then

$w_{i+1}=w_{i}+\alpha\left(x-w_{i}\right)$

If the winner does not belong to the correct class, then

$w_{i+1}=w_{i}-\gamma\left(x-w_{i}\right)$ where $w_{i+1}$ is used to represent the weight vector after adjustment, $w_{i}$ the vector before adjustment, $\alpha$ and $\gamma$ are learning parameters. The algorithm is briefly described as below:

For each input sample, the two closet weights vectors $w_{i}$ and $w_{j}$ are first found by using the Euclidean distance criterion. Assume the distances from $w_{i}$ and $w_{j}$ to $x$ are $d_{i}$ and $d_{j}$, respectively. If the two closest weight vectors belong to different classes, one of them is correct, say, $x$ and $w_{i}$ represent different classes. Furthermore, when the input sample $x$ be found in the windows between the two closest weight vectors, then [11]:

$$
\begin{aligned}
& w_{i}(t+1)=w_{i}(t)-\alpha(t)\left(x-w_{i}(t)\right) \\
& w_{j}(t+1)=w_{j}(t)+\alpha(t)\left(x-w_{j}(t)\right)
\end{aligned}
$$

Let $t$ represent the number of training set iterations. The window could be also defined in terms of relative distances $d_{i}$ and $d_{j}$ from $w_{i}$ and $w_{j}$ to $x$, respectively, having a constant ratio $s$. Then the input vector $x$ is defined to be contained the windows if $\min \left(d_{i} / d_{j}, d_{j} / d_{i}\right)>s$, with $s=(1-w) /(1+w)$.

\section{EXPERIMENTAL AND ANALYSIS}

\subsection{The Process of Wavelet Packet}

Experiments were conducted in order to validate the approach, which is developed in this article. Figs. (3, 6, 9 and 12) show time domain signals of the railway rolling bearing. Fig. (4, 7, 10 and 13) show the de-nosing signal. Fig. (5, 8, 11 and 14) show the de-nosing signal decomposed with wavelet packet. 


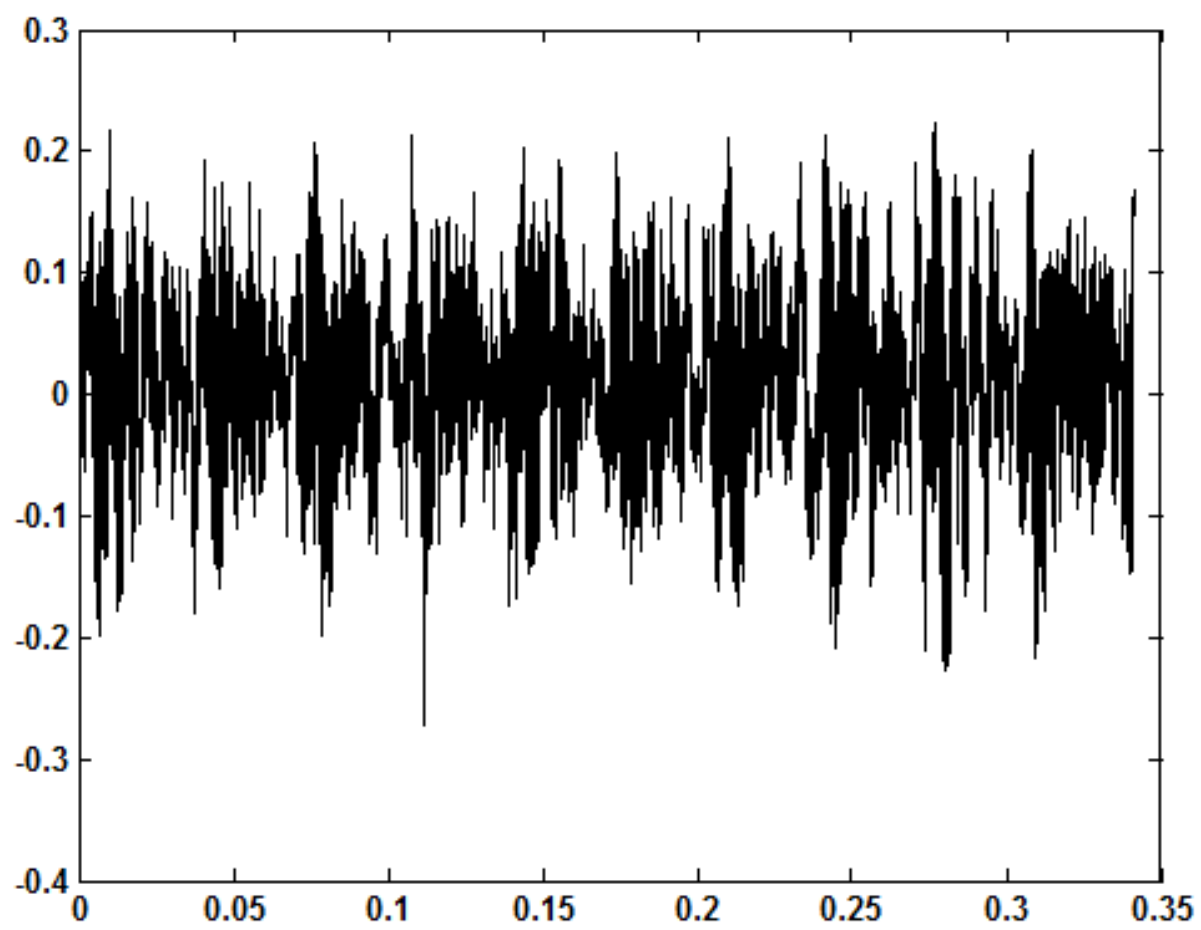

Fig. (3). Vibration signal of a normal roller bearing.

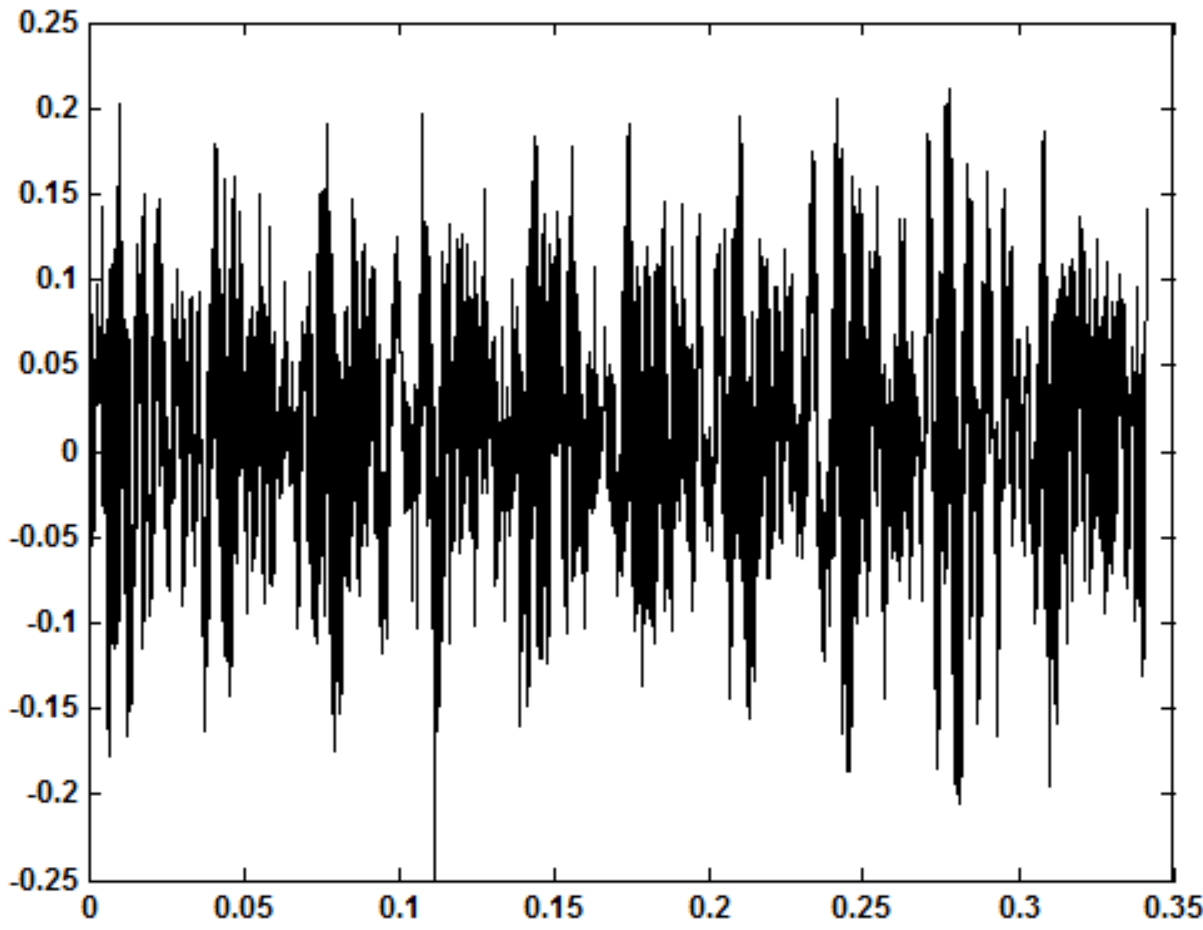

Fig. (4). The de-nosing signal of normal roller bearing. 

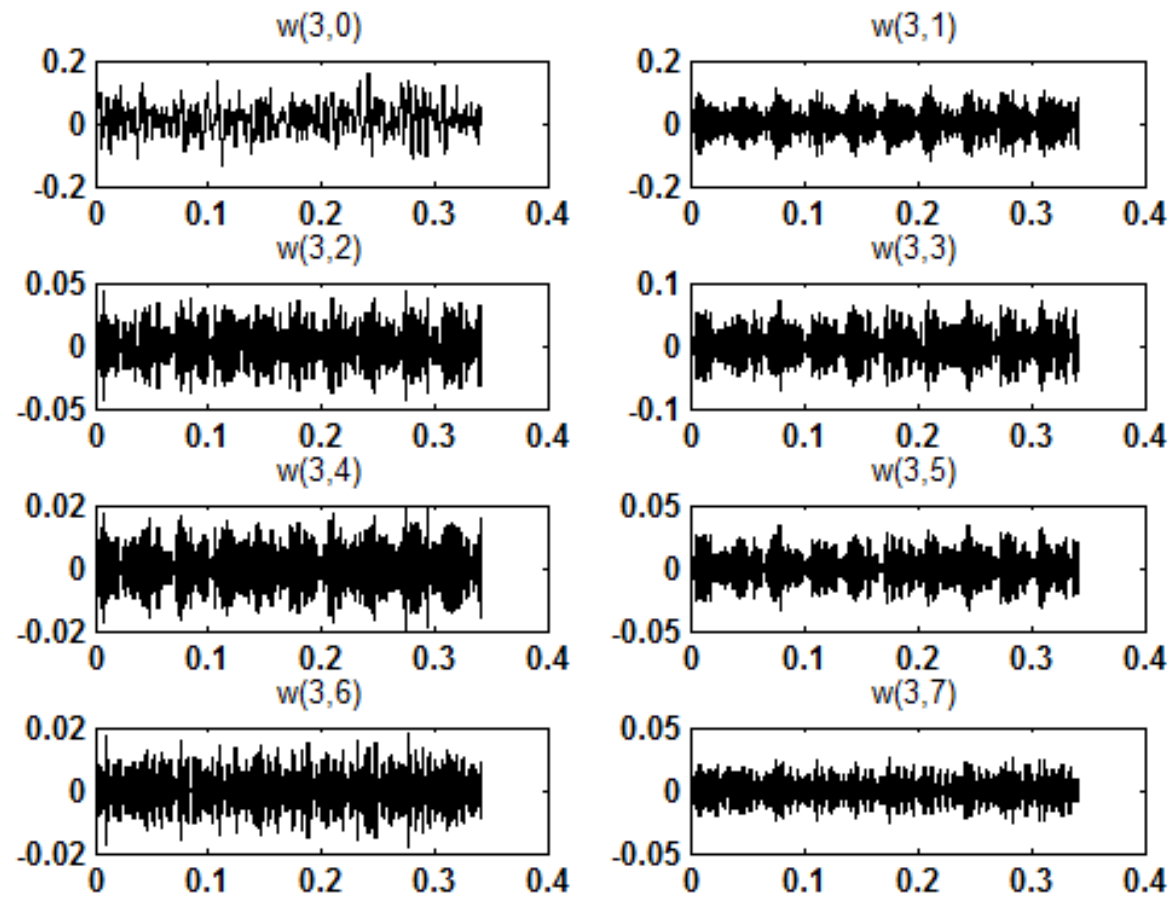

Fig. (5). The de-nosing normal signal decomposed with wavelet packet.

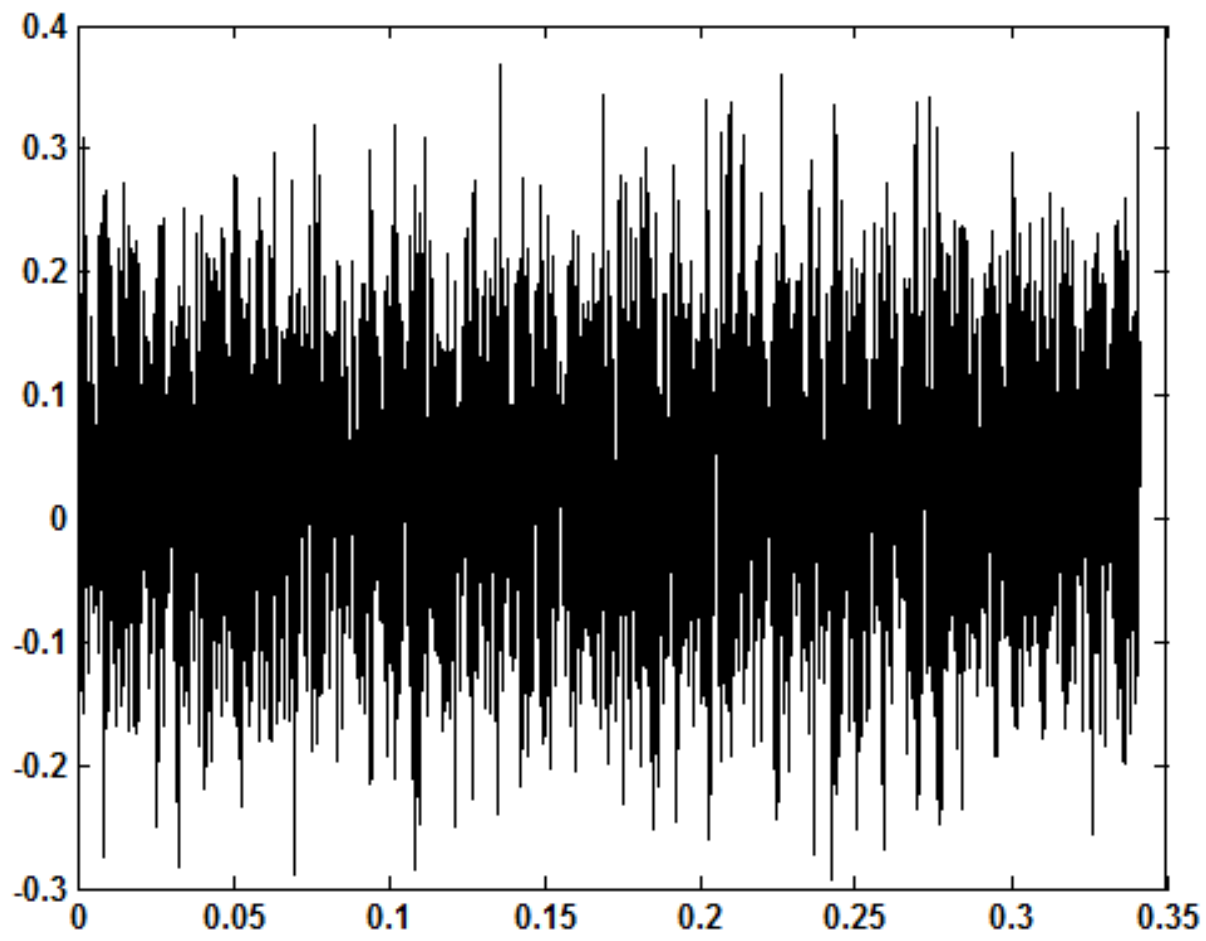

Fig. (6). Vibration signal of a roller bearing with rolling fault. 


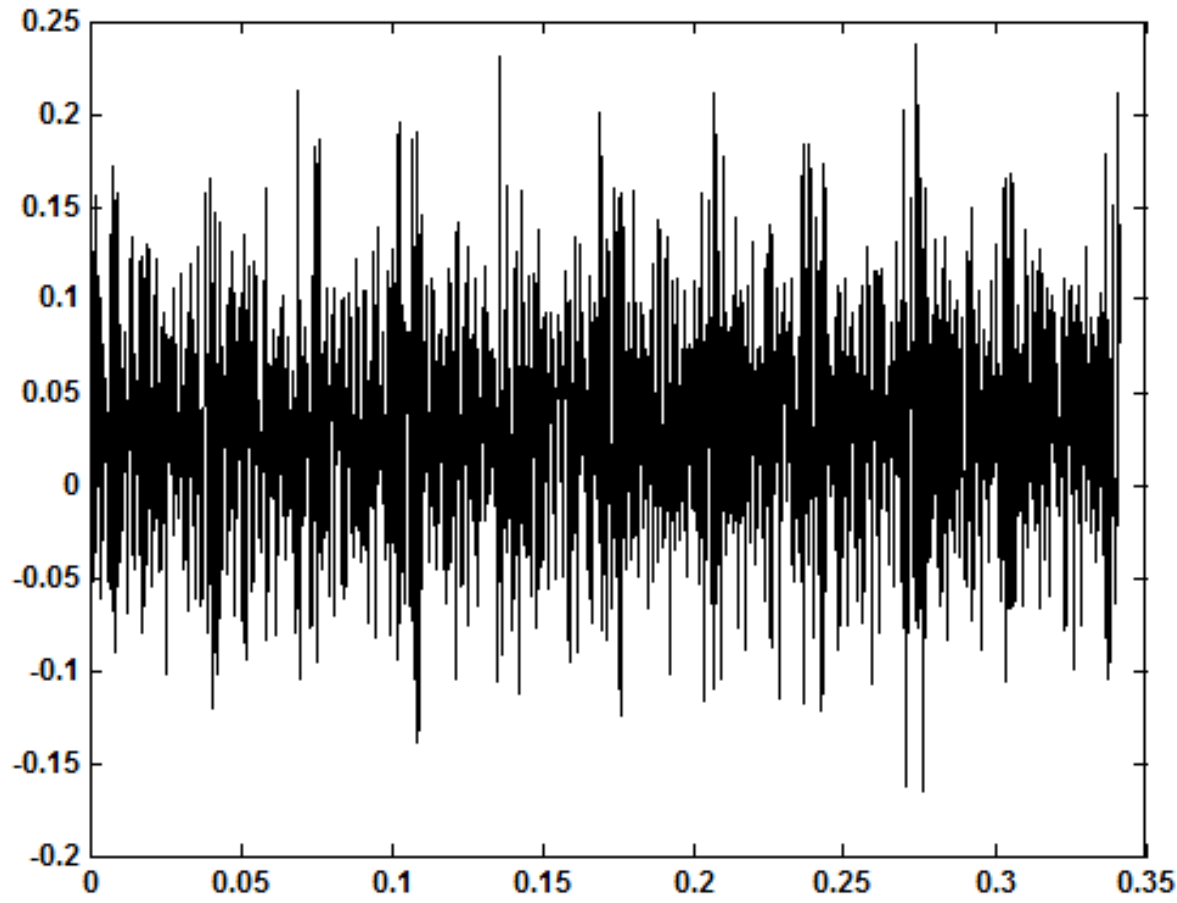

Fig. (7). The de-nosing signal of rolling fault bearing.
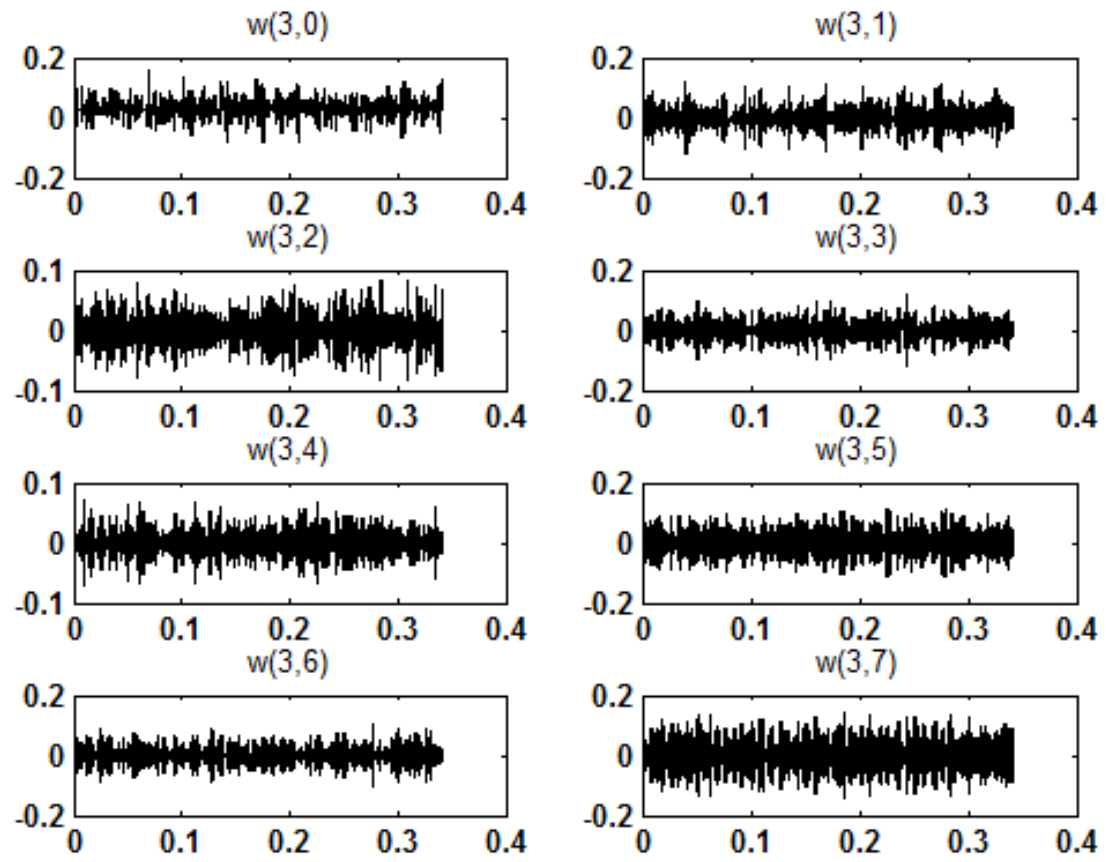

Fig. (8). The de-nosing rolling fault signal decomposed with wavelet packet.

Three-layer wavelet packet is adopted to decompose the signal of railway rolling bearings, and then the wavelet packet energy eigenvector was constructed. Next, energy of each band is got, so part of the normalized training data is listed in Table 1. Part of the testing data is listed in Table 2.

\subsection{The Process of Neural Network}

The fault diagnosis process of railway rolling bearing based on LVQ neural network is as follows:

(1) Determining the input vector of the LVQ neural network. Making 8 extracted feature parameters as the fault feature vectors and making them as the input vectors of the LVQ neural network. 


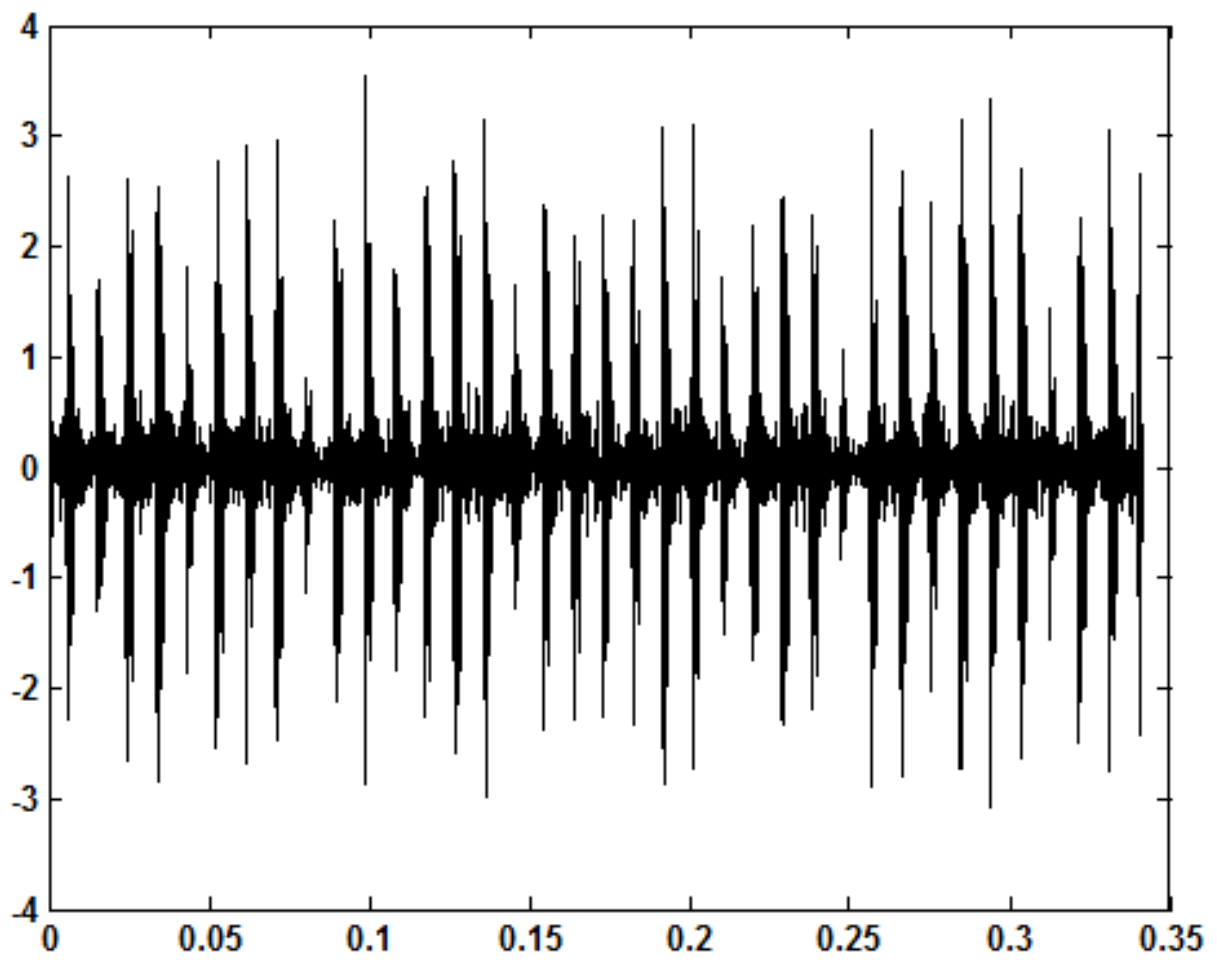

Fig. (9). Vibration signal of a roller bearing with outer ring fault.

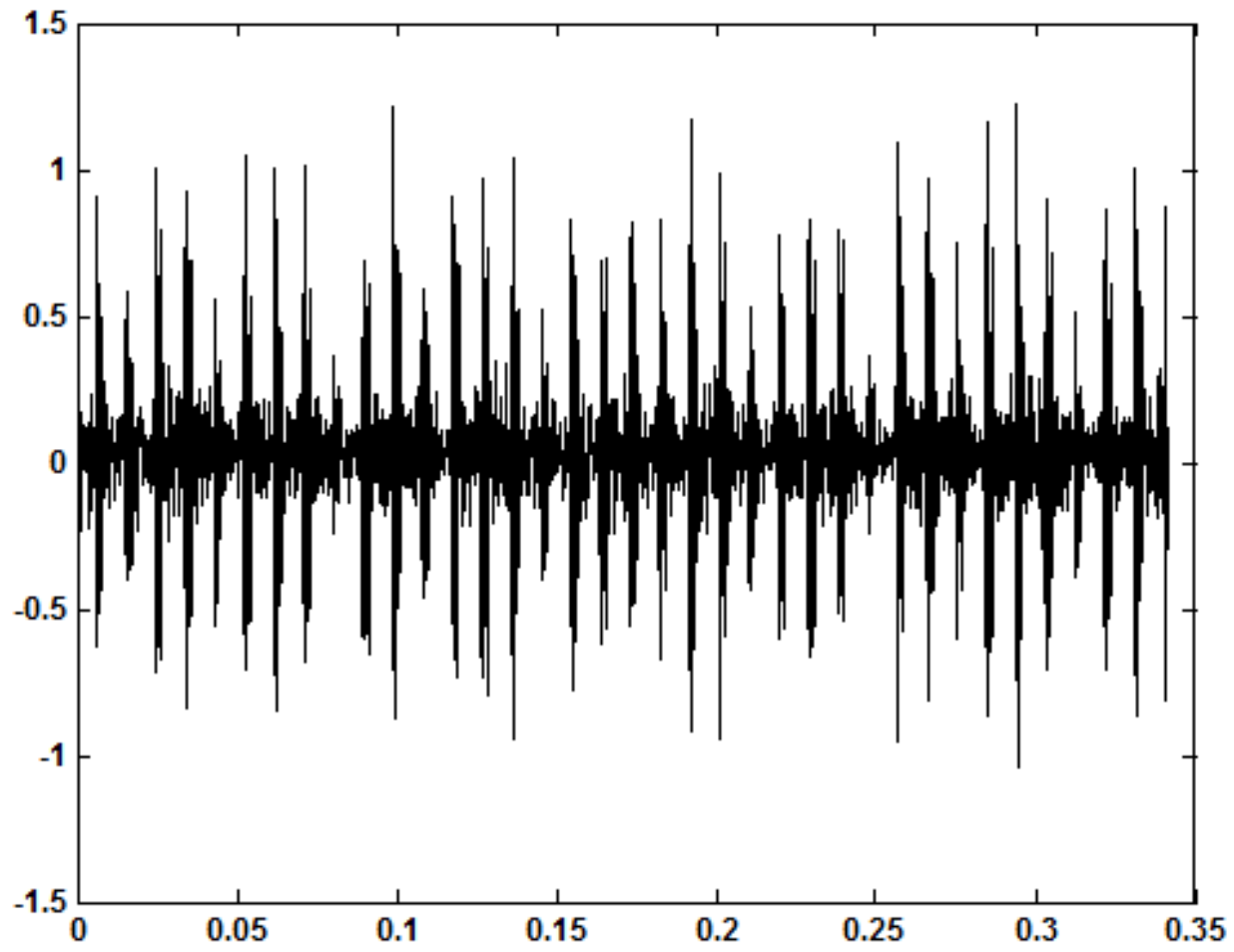

Fig. (10). The de-nosing signal of outer ring fault bearing. 

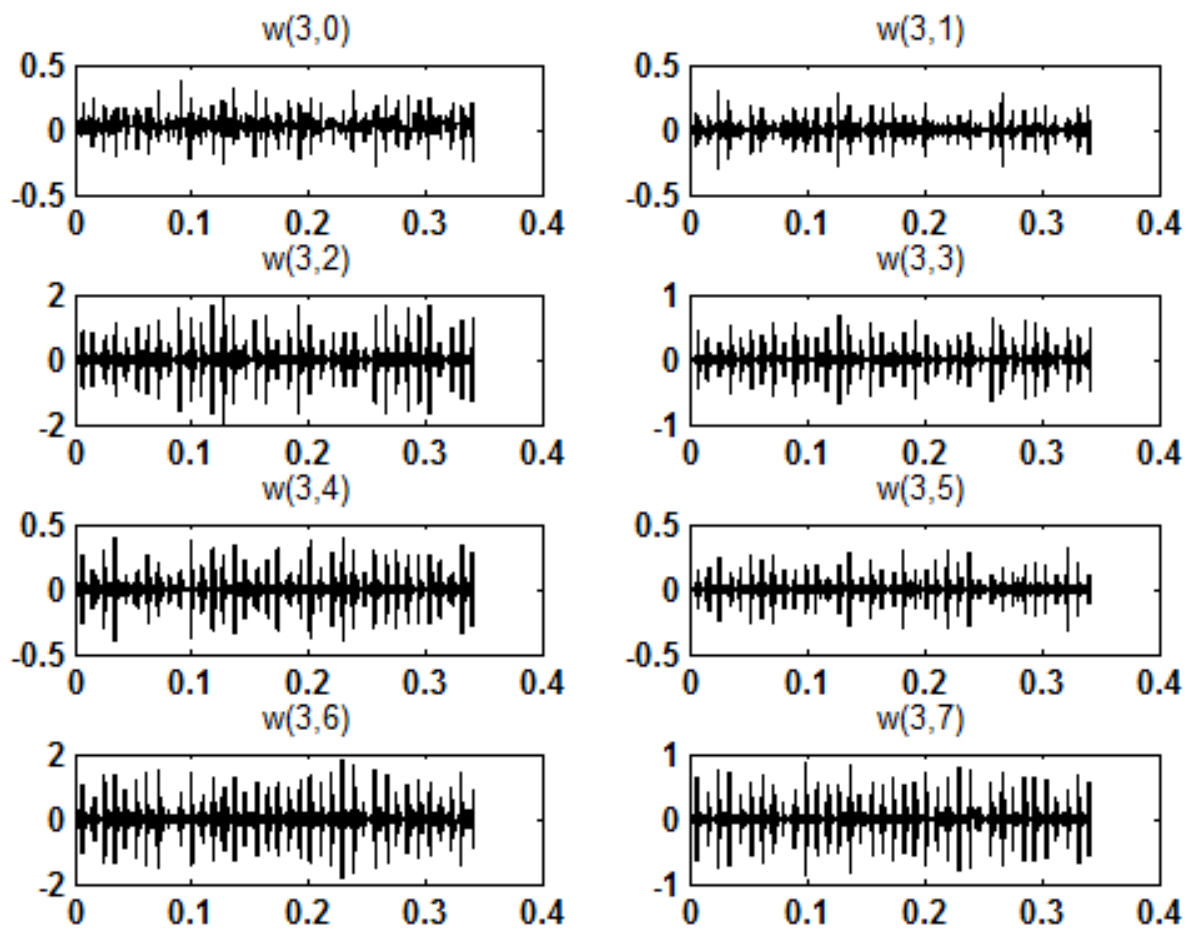

Fig. (11). The de-nosing outer ring fault signal decomposed with wavelet packet.

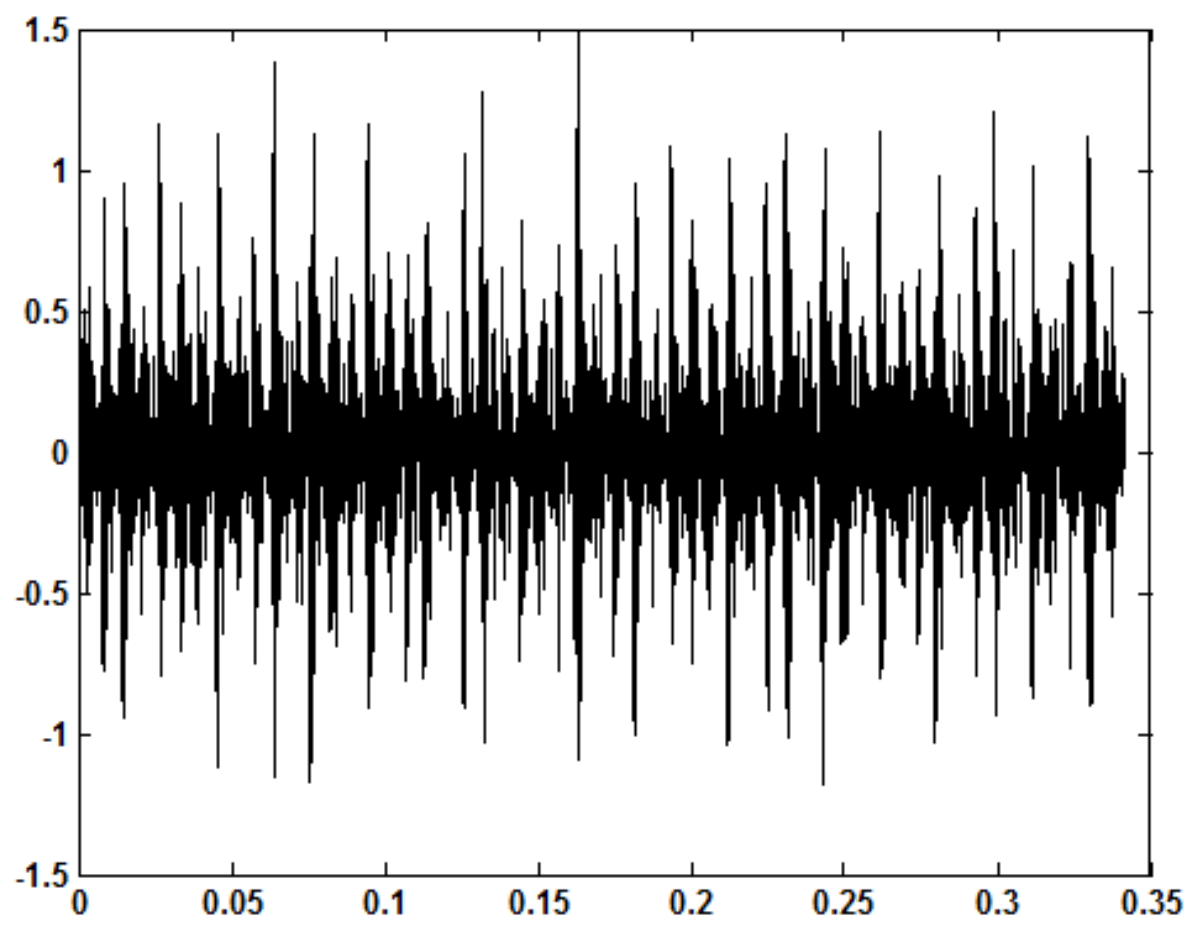

Fig. (12). Vibration signal of a roller bearing with inner ring fault. 


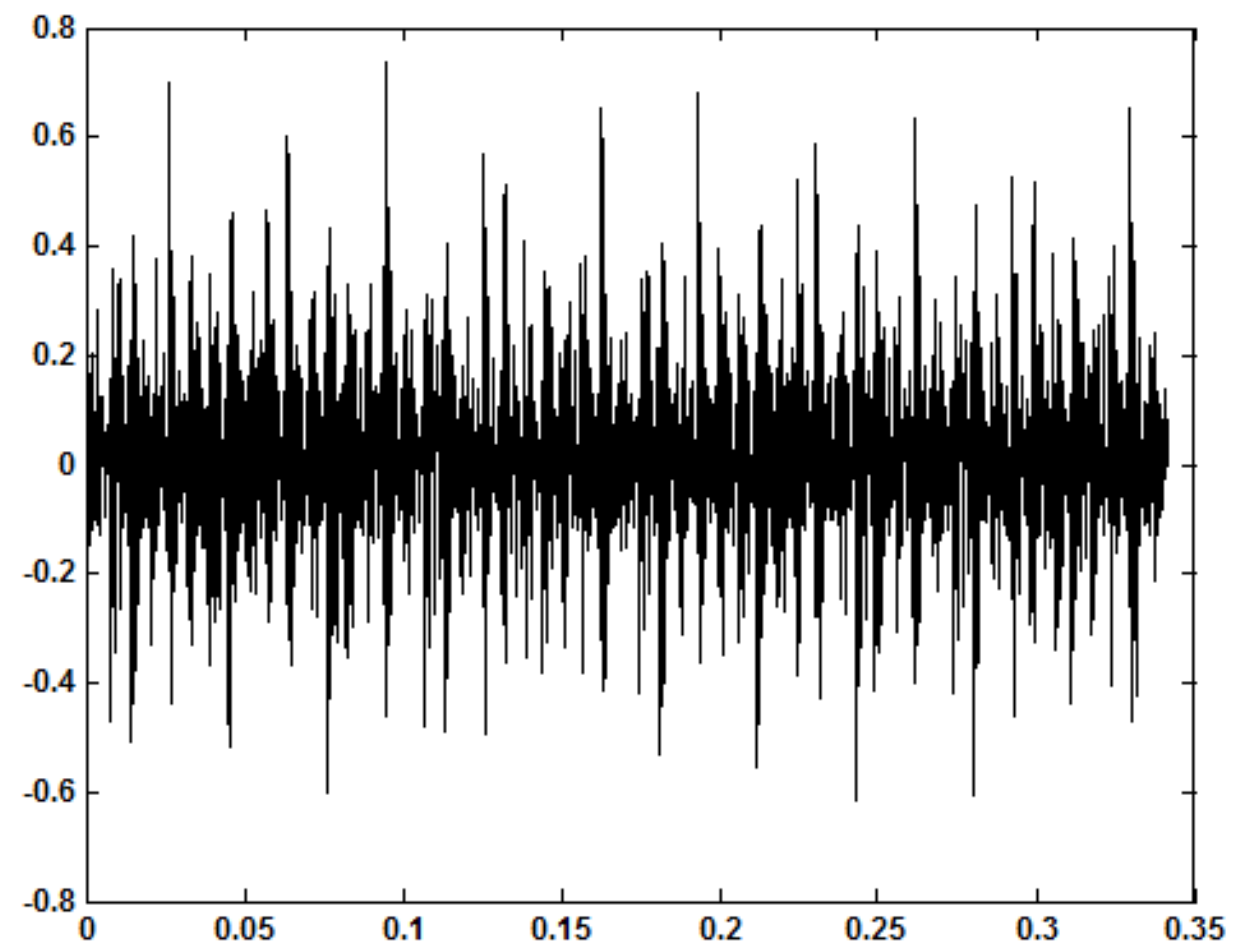

Fig. (13). The de-nosing signal of inner ring fault bearing.
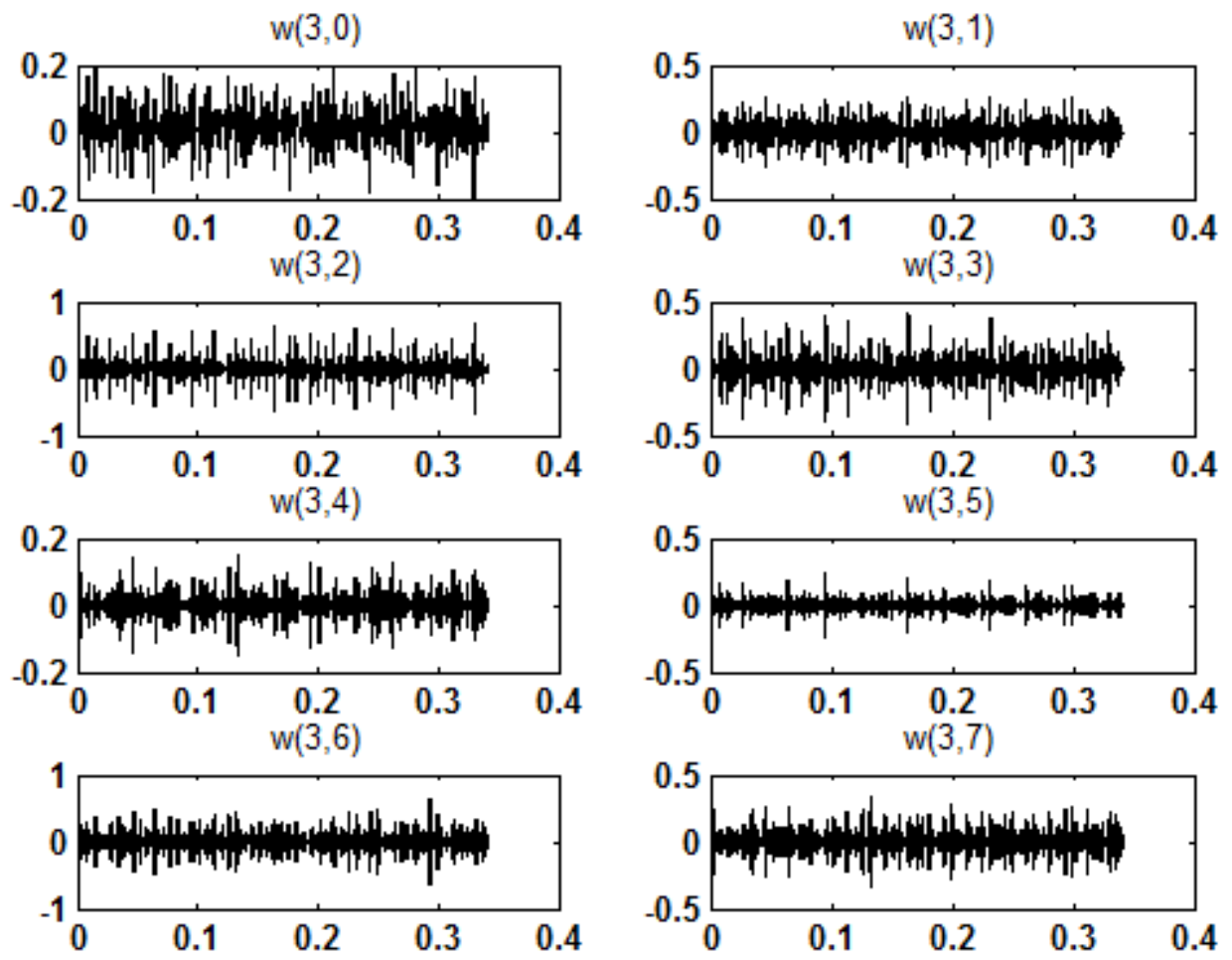

Fig. (14). The de-nosing inner ring fault signal decomposed with wavelet packet.

(2) Coding the fault types of railway rolling bearing. The output of LVQ neural network corresponds to different fault types of the roller bearing, the expected output for normal bearing is 1 , the expected output for outer circle fault bearing is 2 , the expected output for inner circle fault bearing is 3 , the expected output for rolling body fault bearing is 4 . 
Table 1. Sample data of bearing operation.

\begin{tabular}{|c|ccccccccccc|}
\hline & \multicolumn{10}{|c|}{ Feature Samples } & Fault Status \\
\hline \hline 1 & 0.4073 & 0.3511 & 0.0450 & 0.1362 & 0.0088 & 0.0278 & 0.0067 & 0.0171 & normal signal \\
\hline 2 & 0.5020 & 0.2460 & 0.0538 & 0.1295 & 0.0087 & 0.0216 & 0.0125 & 0.0261 & normal signal \\
\hline 3 & 0.3400 & 0.3855 & 0.0506 & 0.1562 & 0.0096 & 0.0319 & 0.0070 & 0.0191 & normal signal \\
\hline 4 & 0.1917 & 0.1166 & 0.0649 & 0.0952 & 0.0436 & 0.1455 & 0.0811 & 0.2615 & rolling fault signal \\
\hline 5 & 0.1875 & 0.1179 & 0.0565 & 0.1108 & 0.0485 & 0.1367 & 0.0854 & 0.2565 & rolling fault signal \\
\hline 6 & 0.1915 & 0.1176 & 0.0679 & 0.0963 & 0.0427 & 0.1383 & 0.0832 & 0.2625 & outer ring fault signal \\
\hline 7 & 0.0191 & 0.0088 & 0.3838 & 0.0602 & 0.0208 & 0.0101 & 0.3976 & 0.0996 & outer ring fault signal \\
\hline 8 & 0.0160 & 0.0111 & 0.3310 & 0.0569 & 0.0243 & 0.0141 & 0.4497 & 0.0969 & outer ring fault signal \\
\hline 9 & 0.0172 & 0.0081 & 0.4027 & 0.0669 & 0.0193 & 0.0150 & 0.3932 & 0.0776 & inner ring fault signal \\
\hline 10 & 0.0564 & 0.0923 & 0.3085 & 0.1276 & 0.0180 & 0.0263 & 0.2732 & 0.0977 & inner ring fault signal \\
\hline 11 & 0.0504 & 0.0909 & 0.2823 & 0.1180 & 0.0182 & 0.0243 & 0.3110 & 0.1049 & inner ring fault signal \\
\hline 12 & 0.0448 & 0.0996 & 0.2724 & 0.1197 & 0.0186 & 0.0229 & 0.3225 & 0.0995 & \\
\hline
\end{tabular}

Table 2. Testing data.

\begin{tabular}{|c|cccccccccc|c|}
\hline & \multicolumn{1}{|c|}{ Feature Samples } & Fault Status \\
\hline \hline 1 & 0.5046 & 0.2403 & 0.0540 & 0.1309 & 0.0083 & 0.0223 & 0.0122 & 0.0273 & normal signal \\
\hline 2 & 0.1730 & 0.1284 & 0.0579 & 0.1075 & 0.0469 & 0.1439 & 0.0763 & 0.2662 & outer ring fault signal \\
\hline 3 & 0.0187 & 0.0083 & 0.3407 & 0.0595 & 0.0241 & 0.0125 & 0.4430 & 0.0931 & inner ring fault signal \\
\hline 4 & 0.0549 & 0.0931 & 0.2513 & 0.1246 & 0.0216 & 0.0238 & 0.3213 & 0.1094 & rolling fault signal \\
\hline
\end{tabular}

(3) Determining the relative parameters of LVQ neural network. The network architecture is usually used for fault diagnosis containing 8 inputs corresponding to the 8 different ranges of the frequency spectrum of a fault signal, 4 outputs corresponding to 4 respective signals, such as normal signal, rolling fault signal, outer ring fault signal and inner ring fault signal and.

(4) Testing network

After training the network, the testing group is used to examine the trained LVQ network.

The results of the examination sample are:

yc_test $=1234$

\section{CONCLUSION}

In this paper, the method of FIR-wavelet packet transform and LVQ neural network is presented to diagnose railway rolling bearings faults based on feature extracting of fault bearing. The proposed technique is robust to practical application. The results show that this method can diagnose the fault of railway rolling bearings. It provides the theoretical foundation for machine fault diagnosis.

\section{CONFLICT OF INTEREST}

The authors confirm that this article content has no conflict of interest.

\section{ACKNOWLEDGEMENTS}

This paper was supported by the national natural science fund project (51175028) and Beijing outstanding talent training projects (2010D005017000007).

\section{REFERENCES}

[1] J.W. Yang, D.C. Yao, and G.Q. Cai, "A method of bearing fault feature extraction based on improved wavelet packet and hilbert analysis", International Journal of Digital Content Technology and its Applications, vol. 4, pp. 127-139, 2010. 
[2] M. Haji, and H.A. Toliyat, "Patern recognition-a technique for induction machines rotor fault detection eccentricity and broken bar fault", Conference Record of the 2001 IEEE Industry Applications Conference, vol. 3, pp. 1572-1578, 2001.

[3] S. Nandi, and H.A. Toliyat, "Condition monitoring and fault diagnosis of electrical machines - a review", IEEE Industry Applications Conference, vol. 1, pp. 197-204, 1999.

[4] G.A. Yang, F.Y. Xu, and Z.H. Wu, "Research on the multi-fault comprehensive diagnosis method based on wavelet packet and demodulation", Journal of Southeast University, vol. 1, pp. 42-45, 2004.

[5] K. Hornik, M. Stinchcombe, and H. White, "Multilayer feedforward networks are universal approximators", Neural Networks, vol. 2, pp. 359-366, 1989.

[6] G.Q. Cai, J.W. Yang, L.M Jia, and D.C. Yao, "Fault diagnosis of railway rolling bearing based on wavelet packet and Elman neural network", Proceedings of the $12^{\text {th }}$ IASTED International Conference Intelligent Systems and control, pp. 268-274, 2009.
[7] Z.M. Ye, B. Wu, and Alireza Sadeghian, "Current signal analysis of induction mechanical faults by wavelet packet decomposition", IEEE Transactions on Industrial Electronics, vol. 6, pp. 1217-1228, 2003.

[8] D.C. Yao, J.W. Yang, and Y.F. Yin, "Fault diagnosis of railway bearing based on muti-method fusion techniques", Machine Design and Research, vol. 3, pp. 70-73, 2010.

[9] M.K. Bashar, N. Ohnishi, T. Matsumoto, Y. Takeuchi, H. Kudo, and K. Agusa, "Image retrieval by pattern categorization using wavelet domain perceptual features with LVQ neural network", Pattern Recognition Letters, vol. 26, pp. 2315-2335, 2005.

[10] A.K. Qin, and P.N, Suganthan, "Initialization insensitive LVQ algorithm based on cost-function adaptation", Pattern Recognition, vol. 38, pp. 773-776, 2005.

[11] J.L. Liu, B.Q. Zuo, X.Y. Zeng, and P. Vroman, "Nonwoven uniformity identification using wavelet texture analysis and LVQ neural network", Expert Systems with Applications, vol. 37, pp. 2241$2246,2010$.

Received: December 15, 2014

Revised: January 04, 2015

Accepted: February 25, 2015

(C) Jianwei et al.; Licensee Bentham Open.

This is an open access article licensed under the terms of the Creative Commons Attribution Non-Commercial License (http://creativecommons.org/licenses/by$\mathrm{nc} / 4.0 /$ ) which permits unrestricted, non-commercial use, distribution and reproduction in any medium, provided the work is properly cited. 